\title{
Cosmological gravitons back reaction and the primordial nucleosynthesis
}

\author{
M. R. G. Maia ${ }^{1}$, J. C. Carvalho ${ }^{1}$, J. S. Alcaniz ${ }^{1}$ and J. M. F. Maia ${ }^{1,2}$ \\ ${ }^{1}$ Departamento de Física-UFRN, CP 1641, 59072-970 Natal, RN-Brasil \\ ${ }^{2}$ Instituto de Física-USP, CP 66318, 05389-970 São Paulo, SP-Brasil
}

\begin{abstract}
The back reaction of effective gravitons created during noninflationary epochs due to the inequivalence of vacuum states at different eras is examined in the context of primordial nucleosynthesis. Our final purpose is to obtain limits on the model employed to study such a process.
\end{abstract}

The inequivalence of vacuum states at different eras of the history of the universe determines the production of gravitational waves in scales larger than the Hubble lengh. During noninflationary periods of expansion, these very long tensor perturbations (VLTP's) enter the Hubble radius, thus becoming effective gravitational waves (EGW's) (Allen 1988). Such an effect adds new contributions to the gravitons energy density $\rho_{g}$ within the horizon, the modes energetically meningful, and can be described by using a macroscopic approach to matter creation (see de Garcia Maia et al., 1997 an references therein). This is done by introducing a creation pressure term in the balance equation for $\rho_{g}$ and the EWG's back reaction leads to the following dynamical equation for the scale factor $a(t)$ during the radiation era (for the flat case):

$$
\frac{\ddot{a}}{a}+\frac{3 \gamma_{r}-2}{2} \frac{\dot{a}^{2}}{a^{2}}=4 \pi G\left(\gamma_{r} \rho_{g}+\frac{\dot{\rho}_{g}}{3 H}\right),
$$

where $H$ is the Hubble parameter, $\gamma_{r}$ is the barotropic parameter related to the dominant component of the era denote by the subscript $r$ and the whole R.H.S. is due to the effective gravitons creation process. It can be shown (de Garcia Maia et al. 1997) that given $\gamma_{r-1}, \gamma_{r}$ and $H\left(t_{r}\right)$, the explicit expression for $\rho_{g}$ is univocally determined in terms of $a(t)$ and $\dot{a}(t)$, so that $a(t)$ can be determined.

Here, we will consider that a transition occurred between a phase denoted by $r=0$, for example an inflationary period, to the radiation era $(r=1)$. In such a case,

$$
\rho_{g}(t)=\frac{1}{\pi^{2}}\left[\frac{\left(3 \gamma_{0}-2\right) H_{1}}{2}\right]^{4}\left[\frac{a_{1}}{a(t)}\right]^{4} I(t),
$$

where $I(t)$ accounts for the graviton creation and is given by (Carvalho et al. 2000)

$$
I(t)=\frac{\left(2 m_{0}+1\right)^{2} \Gamma^{2}\left(-m_{0}\right)}{\pi\left(2 m_{0}+3\right) 2^{2 m_{0}+5}}\left[1-\left(\frac{4 \pi}{a_{1} H_{1}\left(3 \gamma_{0}-2\right)} \dot{a}(t)\right)^{2 m_{0}+3}\right],
$$




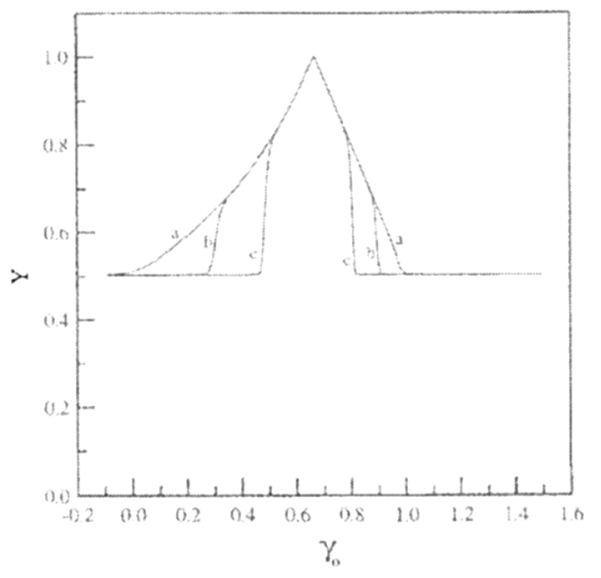

Figure 1. The asymptotic value of the slope $Y=\left[d \operatorname{loga}(t) / a_{1}\right][\operatorname{dlog} t]$ as a function of the barotropic parameter $\gamma_{o}$. Curves (a), (b), and (c) correspond to $\left(H_{1} / \mathrm{m}_{\text {planck }}\right)=1,\left(H_{1} / \mathrm{m}_{\text {planck }}\right)=10^{-5},\left(H_{1} / \mathrm{m}_{\text {planck }}\right)=$ $10^{-10}$, repectively. As can be seen, as $\gamma_{o}$ gets close to $2 / 3$, the slope $Y$ changes drastically from the standard value $1 / 2$ to peak at the value 1 for $\gamma_{o}=2 / 3$.

with $m_{r} \equiv 2 /\left(3 \gamma_{r}-2\right)-1 / 2$. For details on obtaining the above equations, see de Garcia Maia et al. 1997.

We should mention that the production of VLTP's is a natural outcome of quantum mechanics and general relativity in the cosmological context. This phenomenon may predict observational effects, depending on the values of free parameters of the model (de Garcia Maia et al. 1999). Here, we are interested in constraining these parameters from a primordial nucleosynthesis analysis.

Since only effective gravitons are produced, and they are not coupled to the other particles at almost all times of the thermal history of the universe, the radiation expands adiabatically, as in the standard picture. The main modification on primordial nucleosynthesis comes from the time-temperature relation, due to the back reaction effect on the expansion rate (Carvalho et al. 2000):

$$
t=2(10.4)^{2} \int_{T}^{\infty} \frac{d T}{T^{3} \sqrt{1+\alpha I(T)}}
$$

where $\alpha=4(10.4)^{4} \frac{8 G H_{1}}{3 \pi}\left(\frac{3 \gamma_{0}-2}{2 T_{1}}\right)^{4}$. We are presently implementing the numerical code to solve the above equation as well as making the necessary modifications on Kawano's code (Kawano 1992) in order to determine the bounds on the parameter $\gamma_{0}$ from nucleosynthesis. Because the dynamics of the Universe is not very sensitive to the value of $H_{1}$ (de Garcia Maia et al. 1997), we expect that for $\gamma_{0} \sim 2 / 3$ the standard results will be considerably affected (see Fig. 1). In 
particular, the present estimates of the ${ }^{4} \mathrm{He}$ abundance could impose constraints on the possible values of $\gamma_{0}$ (Carvalho et al. 2000).

\section{References}

Allen, B. 1988, Phys. Rev. D, 37, 2078.

Kawano, L. 1992, Fermilab preprint PUB-92/04-A (unpublished)

de Garcia Maia, M. R., Carvalho, J. C. and Alcaniz, J. S. 1997, Phys. Rev. D, $50,6351$.

de Garcia Maia, M. R., Carvalho, J. C. and Alcaniz, J. S. 1999, Phys. Rev. D, $60,123510$.

Carvalho, J. C., de Garcia Maia, M. R., Alcaniz, J. S. and Maia, J. M. F. 2000, in preparation. 